\title{
Laboratory experiments on the interaction between inclined negatively buoyant jets and regular waves
}

\author{
Simone Ferrari ${ }^{1, \mathrm{a}}$ and Giorgio Querzoli ${ }^{1}$ \\ ${ }^{I}$ DICAAR (Dipartimento di Ingegneria Civile, Ambientale e Architettura), University of Cagliari, via Marengo 2, 09123 \\ Cagliari, Italy
}

\begin{abstract}
In this paper we present the results from a series of laboratory experiments on inclined negatively buoyant jets released in a receiving environment with waves. This simulates the case, typical of many practical applications, of the sea discharge of fluids denser than the receiving environment, as in the case of the brine from a desalination plant. The experiments were performed employing a Light Induced Fluorescence (LIF) technique, in order to measure the concentration fields. Both the jet and the wave motion features were varied, in order to simulate a typical discharge into the Mediterranean Sea. Reference discharges in a stagnant environment were performed as well. The jet behaviour was analyzed from a statistical point of view, both considering the global phenomenon and its single phases. The influence of the wave motion on the inclined negatively buoyant jet geometry and dilution turns out to be a combined action of a split into two branches of the jet and a rotation. Their combined action decreases the jet maximum height and the impact distance, and is the main cause for the higher dilution reached in a wavy environment.
\end{abstract}

\section{Introduction}

Inclined Negatively Buoyant Jets (INBJs) develop when a fluid is released, with an angle to the horizontal different from $0^{\circ}$ or $90^{\circ}$, in a less dense fluid environment, as in the case of brine discharges from desalination plants, dense effluents from wastewater treatment plants, etc. This phenomenon has been a topic for many researches during last years (e.g., Besalduch et al. 2014 [1], Jiang et al. 2014 [2], Besalduch et al. 2013 [3], Oliver et al. 2013 [4], Lai and Lee 2012 [5], etc.).

Since Chin in 1987 [6] measured that the dilution in a wavy environment is significantly higher than in a stagnant one, the interaction between wave motion and jets have been largely investigated and discussed (e.g., Ryu et al. 2005 [7], Chang et al. 2009 [8], Hsiao 2011 [9], etc.) but only in few cases buoyant jets were employed (e.g. Chin 1988 [10], Lin et al. 2013 [11]) and the released jets were always vertical or horizontal.

As, to the best of authors' knowledge, the interaction between INBJs and waves was never investigated before, we have carried out an experimental campaign in a flume furnished with a wavemaker, simulating some typical waves of the Mediterranean Sea (matching the geometric, kinematic and dynamic similarity), using data coming from the wave buoy of Alghero (North-West Sardinia). The interaction between these waves and INBJs, with two different densimetric Froude numbers, and the same Reynolds number, was investigated in a laboratory model

\footnotetext{
${ }^{\mathrm{a}}$ Corresponding author: ferraris@unica.it
}

by means of Image Analysis techniques, to measure concentration and velocity fields. Here we present concentration data, acquired by means of a Laser Induced Fluorescence (LIF) technique, non-intrusive and able to measure the concentration all over the investigation field. Reference discharges in a stagnant environment were performed too, to allow a comparison with the ones biased by waves. INBJ behaviour was statistically analyzed, taking into account the global phenomenon and its single phases, in order to better understand the influence of wave parameters on the jet geometry and dilution.

In order to introduce the qualitative behaviour of a simple jet released in a receiving body affected by waves, an essential literary review on the topic of interaction between waves and simple jets (vertical or horizontal) is hereafter presented.

Three different regions were spotted by Hwung and Chyan in 1993 [12], namely, starting from the simple jet origin:

1) jet deflection region (JDR);

2) transition region (TR);

3) developed jet region (DJR).

In the JDR, a simple jet is dominated by its initial momentum, consequently preserving its shape and "rigidity"; as in this region the simple jet velocity and the receiving body one (predominantly horizontal, as we are close to the bottom) are of the same magnitude, the wave influence on the jet to force it to oscillate almost like a 
rigid body. This is due to the fact that, close to the jet origin, the transversal dimension of the jet is relatively small compared to the wavelength, so there is not a large variation of the wave horizontal velocity and all the points of the jet are forced, at that instant, in the same direction. Mossa in 2004 [13] found the same result from its Laser Doppler Anemometry (LDA) measurements. Other Authors (e.g. Sharp 1986 [14], Chyan et al. 1991 [15]) have found that the oscillatory motion induced on simple jets by a regular wave motion considerably affects the dilution achieved by a simple jet: this because the zone of flow establishment (where the velocity profile changes from the top-hat profile to the Gaussian one) is much shorter and the concentration profiles are no more Gaussian but flat-topped or bi-peaked [12]. In the DJR, a periodic deflection has been observed by Hwung and Chyan in 1993 [12] on vertical simple jets and by Chin in 1987 [16] on horizontal ones; they have been found that this deflection is one of the reason for the higher dilution, because large volumes of environment fluid are trapped into the jet fluid above the deflection region during the following deflection (wave traction mechanism, which sums itself to the jet entrainment mechanism).

\section{Experiments and data elaboration}

The experimental configuration (see Figure 1) reproduces, in a flume, a typical submarine outfall: a portion of pipe laid down on the sea floor, releasing the effluent from a sharp-edge orifice on its lateral wall. An in-depth description of the adopted set-un is reported in Ferrari and Querzoli 2010 [17]. Here, in addition, a piston type wavemaker installed on one side of the flume is used, while on the opposite side an absorbing beach minimizes the wave reflections. The experimental set-up and the run configurations were chosen to respect the geometric, kinematic and dynamic similarity with a real Mediterranean sea discharge of a fluid denser than the sea water, at a scale model $\mathrm{k}_{\mathrm{L}}$ defined by:

$$
k_{L}=1 / 40
$$

INBJs were released with an angle to the horizontal $\theta$ of $67^{\circ}$, which is close to the optimum value for assuring the maximum effluent dilution according to Ferrari and Querzoli 2004 [18].

Simulations were performed with a Reynolds number Re around $10^{3}$, so larger than the critical one (i.e. $5 \cdot 10^{2}$ for the adopted experimental set-up), assuring the jet's sudden transition to turbulence as well as the independence on initial conditions. As documented in literature (List 1979 [19]), the other non-dimensional parameter responsible for the INBJ behaviour is the densimetric Froude number $\mathrm{Fr}$, that controls the ratio between inertial and buoyant forces. Two different Fr values, typical of brine discharges from desalination plants, were considered (28.1 and 17.6) and for each Fr a set of runs with different wave parameters were simulated. Moreover, in order to allow a comparison with the same INBJ released in a wavy receiving body, for each considered $\mathrm{Fr}$ a simulation in a stagnant receiving body was carried out. Table 1 sums up the characteristic parameters set for the experiments. For each analyzed Fr, the ratio between the water depth $d$ and the wavelength $\mathrm{L}$ falls in the deep water regime for the first run $(\mathrm{d} / \mathrm{L}=$ 1.03 ) and in the intermediate-depth water waves one for the remaining cases $(\mathrm{d} / \mathrm{L}=0.23,0.11)$; while the ratio between the wave height $\mathrm{H}$ (equal to $2 \cdot \mathrm{A}$ ) and $\mathrm{L}$ falls for all the runs in the long wave regime. Figure 1 displays experimental data recorded by the Sistema Ondametrico Nazionale - National Wavemetric System - from 2003 to 2005 from the wave buoy of Alghero, in the NorthWestern Sardinia.

This range of data was used in order to choose the laboratory wave parameters with the aim of simulating some typical Mediterranean waves, with respect to the kinematic similarity., granted by the following equations:

$$
\begin{aligned}
& A_{\text {MODEL }}=A_{\text {PROTOTYPE }} \sqrt{k_{L}} \\
& T_{\text {MODEL }}=T_{\text {PROTOTYPE }} \cdot k_{L}
\end{aligned}
$$

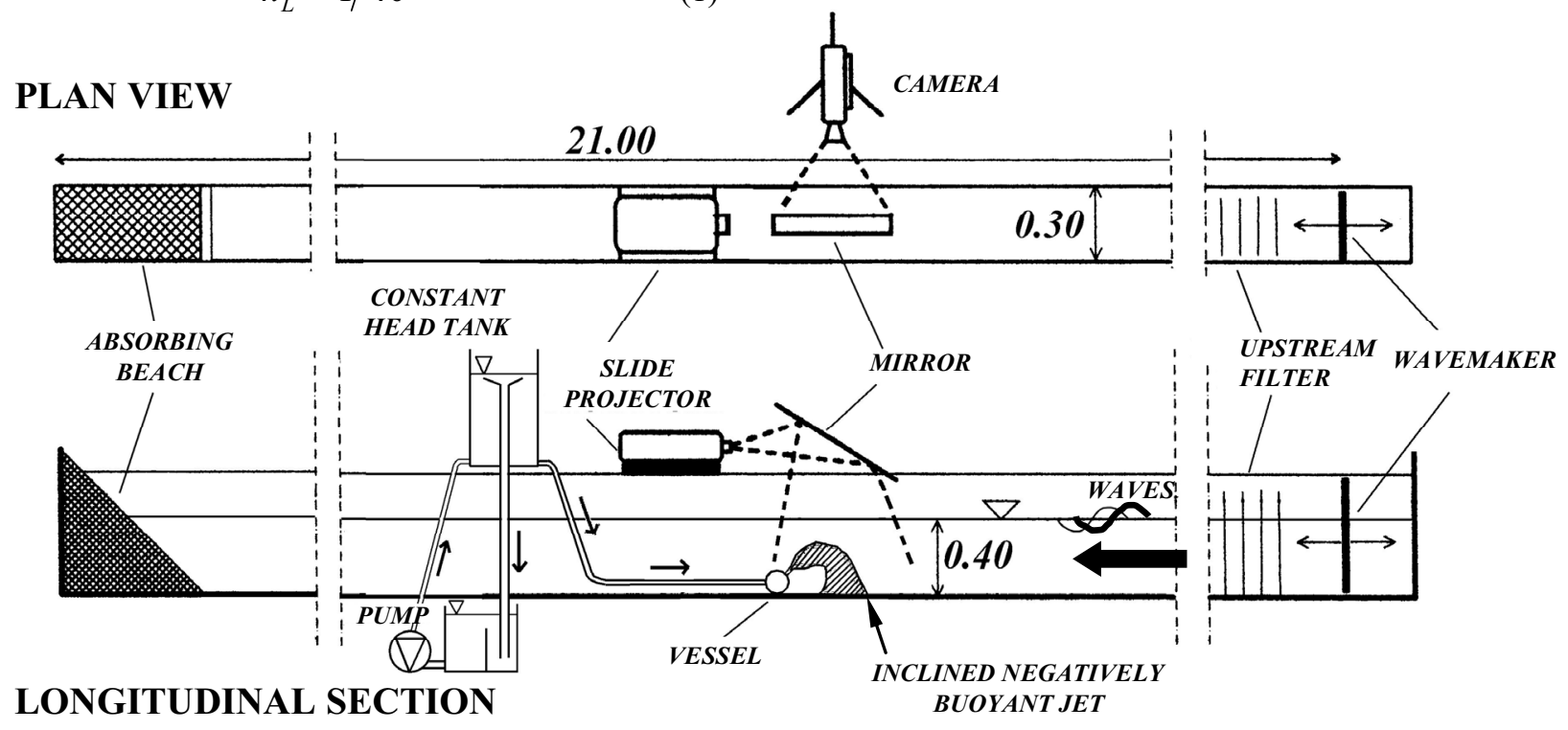

Figure 1. A sketch of the experimental set-up; the Inclined Negatively Buoyant Jets issue from the vessel, simulating a pipe laid down the sea bottom, and the waves, travelling from the right to the left, are generated from the wavemaker; measures are in meters. 
Table 1. Characteristic parameters of the experimental runs; $\mathrm{T}$ is the wave period, $\mathrm{A}$ is the wave amplitude, $\mathrm{N}$ is the number of waves, L is the wave length, $\mathrm{d}$ is the water depth, equal to $0.40 \mathrm{~m} ; \mathrm{Fr}$ is the densimetric Froude number.

\begin{tabular}{|c|c|c|c|c|c|c|c|}
\hline Run & $\mathrm{T}[\mathrm{s}]$ & $\mathrm{A}[\mathrm{mm}]$ & $\mathrm{N}$ & $\mathrm{L}[\mathrm{m}]$ & $\mathrm{d} / \mathrm{L}$ & $2 \mathrm{~A} / \mathrm{L}$ & Fr \\
\hline 1 & 0.50 & 5.00 & 360 & 0.39 & 1.03 & 0.0256 & 28.1 \\
\hline 2 & 1.05 & 5.00 & 170 & 1.72 & 0.23 & 0.0058 & 28.1 \\
\hline 3 & 1.05 & 12.50 & 170 & 1.72 & 0.23 & 0.0145 & 28.1 \\
\hline 4 & 1.50 & 5.00 & 120 & 3.51 & 0.11 & 0.0028 & 28.1 \\
\hline 5 & 1.50 & 12.50 & 120 & 3.51 & 0.11 & 0.0071 & 28.1 \\
\hline 6 & \multicolumn{6}{|c|}{ Stagnant receiving body } & 28.1 \\
\hline 7 & 0.50 & 5.00 & 360 & 0.39 & 1.03 & 0.0256 & 17.8 \\
\hline 8 & 1.05 & 5.00 & 170 & 1.72 & 0.23 & 0.0058 & 17.8 \\
\hline 9 & 1.05 & 12.50 & 170 & 1.72 & 0.23 & 0.0145 & 17.8 \\
\hline 10 & 1.50 & 5.00 & 120 & 3.51 & 0.11 & 0.0028 & 17.8 \\
\hline 11 & 1.50 & 12.50 & 120 & 3.51 & 0.11 & 0.0071 & 17.8 \\
\hline 12 & \multicolumn{6}{|c|}{ Stagnant receiving body } & 17.8 \\
\hline
\end{tabular}

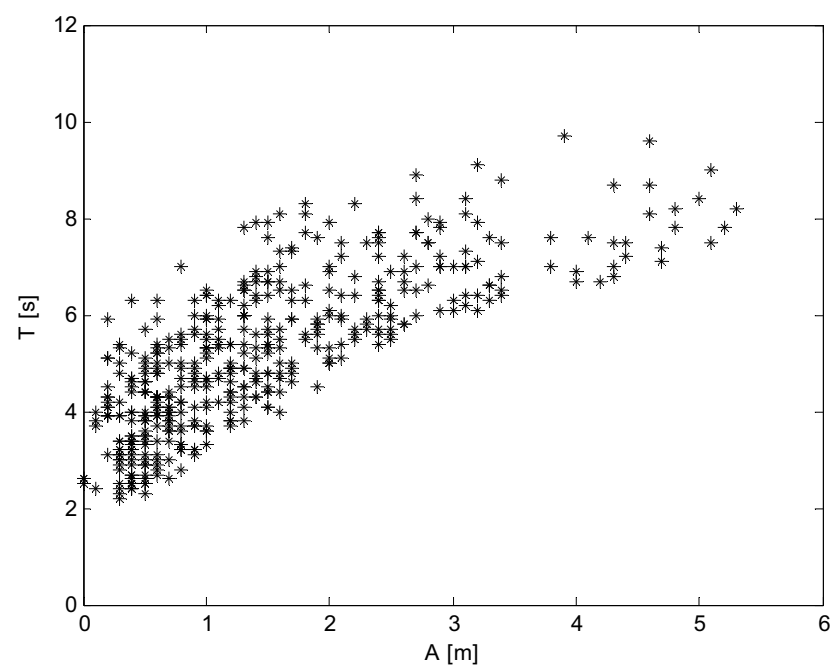

Figure 2. Data of wave amplitude (A) and period (T) recorded by the Sistema Ondametrico Nazionale - National Wavemetric System - from 2003 to 2005 in the wave buoy of Alghero (North-Western Sardinia).

In the performed experiment, the released solution is a mixture of water, sodium-sulphate (to increase density and achieve the prescribed Fr) and a fluoresceine (to allow jet visualization). The axial vertical plane of the INBJ is illuminated from a light sheet obtained from two aligned slide projectors, while a 3-CCD video-camera, placed orthogonal to the light sheet, captured images at the frequency of $25 \mathrm{~Hz}$, with a resolution of $720 \times 576$ pixels and with a bit depth of 8 bit per colour. In Figure 3 , two snapshots of an instantaneous picture of an INBJ with $\mathrm{Fr}=28.1$, released in a stagnant environment $(2 \mathrm{a})$ and in a wavy one with $\mathrm{T}=1.05 \mathrm{~s}$ and $\mathrm{A}=12.5 \mathrm{~mm}(2 \mathrm{~b})$, are shown.

Acquired images were analyzed by means of LightInduced Fluorescence (LIF) in order to measure the instantaneous concentration fields, which were assumed proportional to the measured light intensity. Under the hypothesis of ergodicity, the statistics of mean value and variance of the concentration field were obtained by time averaging (see, for example, Figure 4 and 5): in all the colour maps the colour changes from blue to red as the corresponding value increases. On the mean concentration fields, the jet axis can be identified as the locus of the maxima of the concentration on several cross sections of the jet, and allows the identification of two characteristic parameters of the INBJ: the point of maximum height (a red asterisk in Figure 3 and 4) and the impact distance (a yellow star in Figure 3 and 4), defined as the one at which the jet axis comes back to the release point height. Distances are non-dimensionalized by the outlet diameter D.

Finally, in order to link observations with phenomena occurring at the oscillating free surface, experiments performed in a wavy environment were also analyzed dividing the wave period in 8 peculiar times (called phases from this point on)

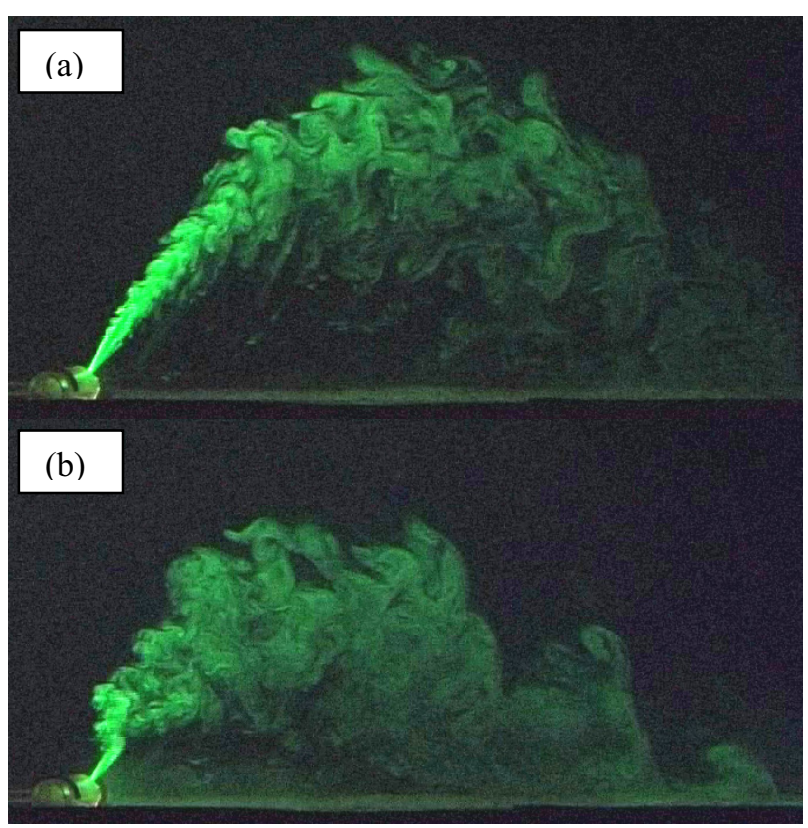

Figure 3. Instantaneous images of an INBJ with $\mathrm{Fr}=28.1$, released with an angle to the horizontal $\theta$ of $67^{\circ}$ in a receiving environment without (a) and with waves (b), with $\mathrm{T}=1.05 \mathrm{~s}$ and $\mathrm{A}=12.5 \mathrm{~mm}$.

\section{Results}

\subsection{Phase averaged concentration fields}

Figure 4 elucidates temporal behaviour of an INJB during the wave passage: it shows the mean concentration fields obtained during each of the 8 phases of a jet with $\mathrm{Fr}=$ 17.8 subjected to a regular wave motion with $\mathrm{T}=1.05 \mathrm{~s}$ and $\mathrm{A}=12.5 \mathrm{~mm}$. Moreover, to make easier the comparison with the stationary case, on each panel the axis (white line) of the same jet released in a still receiving body (whose mean concentration field is shown on Figure 10a) is superimposed, while a red asterisk and a yellow star highlight, respectively, the maximum height point and the impact point.

Looking at Figure 4, it is possible to see how the INBJ is deflected in its initial stage (compare the jet concentration field and the axis, which is relative to the stagnant case): it has more momentum and so tends to 
preserve its initial direction or, in other words, to maintain its "rigidity". As stated before, in agreement with [12], this region corresponds to the jet deflection region (JDR) identified in simple jets. Moreover, the oscillation is larger in the upward direction than in the downward one: this is possibly due to the velocity field generated by the interaction between the wave motion (basically horizontal in that region) and the cylindrical vessel simulating the pipe: the velocities are larger on the upper extremity of the vessel and slower in the lower one. As a consequence, the deflection pushing the INBJ upwards tends to carry it towards regions with higher velocity, causing larger displacements.

Moving along the INBJ axis in Figure 4, it is possible to see how, surpassed the JDR, the jet is more deeply deflected upwards or downwards, according to wave phase: the INBJ is in the transition region (TR) where it, slowed down by the interaction with the waves and by the entrainment, is more subject to the wave motion action. In the TR, if T and A are large enough, the INBJ is split into two branches, one higher and one lower than the stationary case, that eventually join in the developed jet region (DJR). This separation in two branches of the INBJ is accentuated by the fact that the change in direction is not gradual but it is abruptly moved from one extreme to the other.

The variance of the concentration field for the same case of Figure 4 is shown in Figure 5 and will be discussed later in paragraph 3.4.

Figure 6 shows the profiles of the mean concentration (normalized by the concentration in the INBJ origin) measured on the point of maximum height (a) and on the impact point (b), in eight different phases, for an INBJ with $\mathrm{Fr}=17.8$ under a regular wave motion with $\mathrm{T}=1.50$ $\mathrm{s}$ and $\mathrm{A}=5.0 \mathrm{~mm}$. $\mathrm{R}$ is the orthogonal distance from the axis: positive values identify the lower region of the INBJ, negative ones the upper region. The bimodal distribution of the concentration maxima strengthens what previously stated on the INBJ alternate displacement following $\mathrm{T}$ and $\mathrm{A}$, in particular on the point of maximum height, where the INBJ bifurcation forces a large oscillation of the jet itself, with big differences among the concentration profiles in the various phases. After this initial instability, in the impact point the various concentration profiles show instead a single peak: this is due to the fact that the INBJ, after the bifurcation experienced in the JDR, tends to become a single entity at the end of the DJR.

Moreover, the concentration profiles on the impact point are similar, pointing out the stabilization of the INBJ and its lost of correlation from the initial conditions, in compliance with the central limit theorem. The effects of Fr on the INBJs at the different phases, is highlighted in Figure 7, where the non-dimensional mean concentration profiles measured on the point of maximum height (for two INBJs with $\mathrm{Fr}=28.1$ and $\mathrm{Fr}=$ 17.8 under a wave motion with $\mathrm{T}=1.05 \mathrm{~s}, \mathrm{~A}=12.5 \mathrm{~mm}$ ) are shown phase by phase.

The above mentioned bimodal distribution is more evident for the heaviest INBJ $(\mathrm{Fr}=17.8)$; moreover, as $\mathrm{Fr}$ decreases, the concentration profiles are narrower. It is interesting to notice also that the concentration profiles tend to be symmetrical, differently from the INBJ in a stagnant environment (see, fro instance, Ferrari and Querzoli 2010 [18]); this is visible in Figure 14 as well, showing the concentration profiles at the impact point, in the case of stagnant (red line) and wavy (blue line) receiving environment.

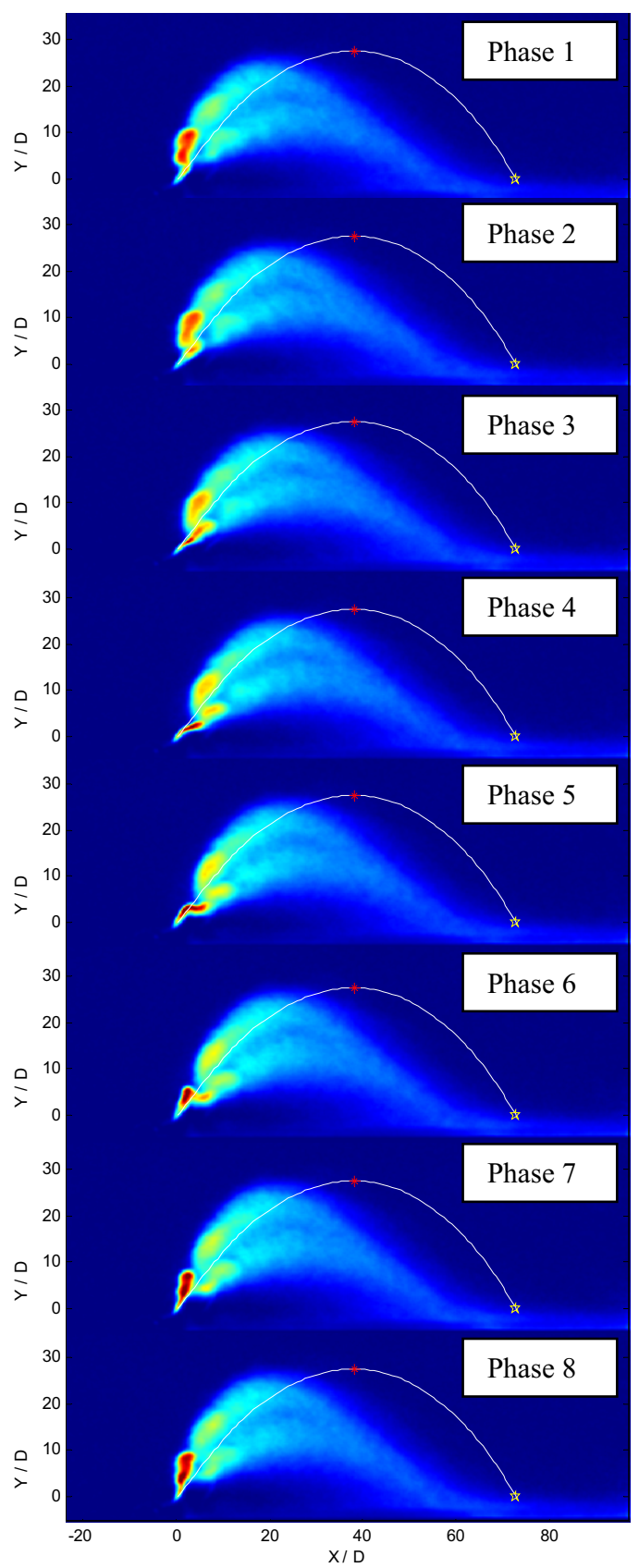

Figure 4. Mean concentration field, phase by phase, of a jet with $\mathrm{Fr}=17.8$, released in a wavy environment with $\mathrm{T}=1.05 \mathrm{~s}$ and $\mathrm{A}=12.5 \mathrm{~mm}$; axes are non-dimensionalized by the outlet diameter D.

The increase in symmetry of the concentration profiles of INBJs in a wavy environment is, most likely, due to the rotation imposed by the wave motion to the released fluid that, when is falling down to the bottom, has lost most of its initial momentum and, consequently, is driven almost only by the negative buoyancy. This rotation induces an additional mixing that accelerate the 
lost of memory of the INBJ initial conditions: this explains why an INBJ in a wavy environment is more symmetrical than the same INBJ released in a still environment.

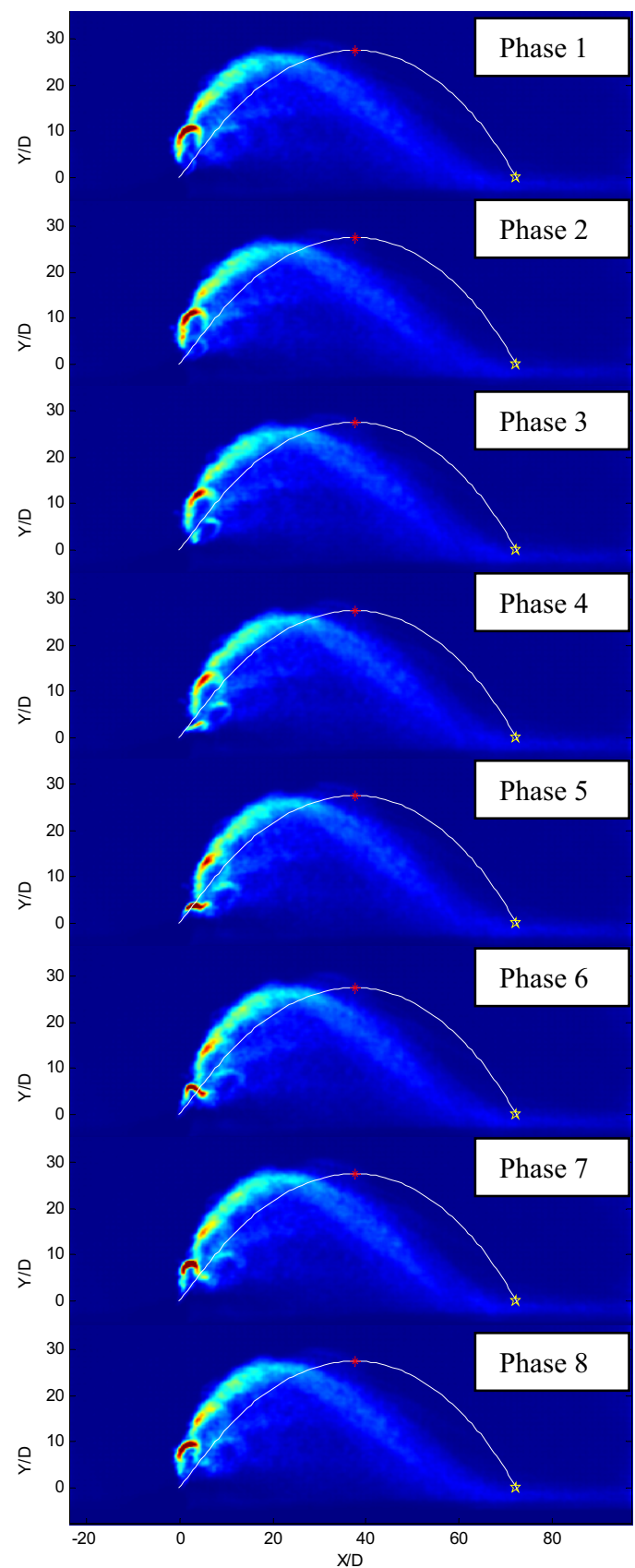

Figure 5. Variance of the concentration field, phase by phase, of a jet with $\mathrm{Fr}=17.8$, released in a wavy environment with $\mathrm{T}=$ $1.05 \mathrm{~s}$ and $\mathrm{A}=12.5 \mathrm{~mm}$; axes are non-dimensionalized by the outlet diameter $\mathrm{D}$

\subsection{Evolution of the point of maximum height and of the impact distance}

As mentioned above, the interaction between waves and an INBJ determines an oscillation of the jet and subsequent split in two branches that thereafter join to a single branch. To deeply investigate this oscillation, on Figure 8 and 9 are shown, respectively, the oscillations of the point of maximum height and of the impact point for the various wave phases, for all the runs with the same Fr.

A simple jet under a wave motion oscillates around its position in stationary conditions (see previously quoted Authors); the point of maximum height and the impact point of INBJs show a different behaviour. Looking at Figure 8, it is possible to see how the point of maximum height never oscillates around its stationary position (the black dash-dotted line) but always tends to remain below it: the higher the wave period $\mathrm{T}$ and amplitude $\mathrm{A}$ are, the lower the maximum height is. If, on one side, it is evident that is the coupling of high $\mathrm{T}$ and $\mathrm{A}$ to bias mainly an INBJ (see the red line for $\mathrm{T}=1.5 \mathrm{~s}$ and $\mathrm{A}=12.5 \mathrm{~mm}$ ), on the other side $\mathrm{T}$ seems to have the biggest role on the decrease of the maximum height: as a matter of fact, the curves in Figure 8 and 9 are sorted by increasing $\mathrm{T}$.
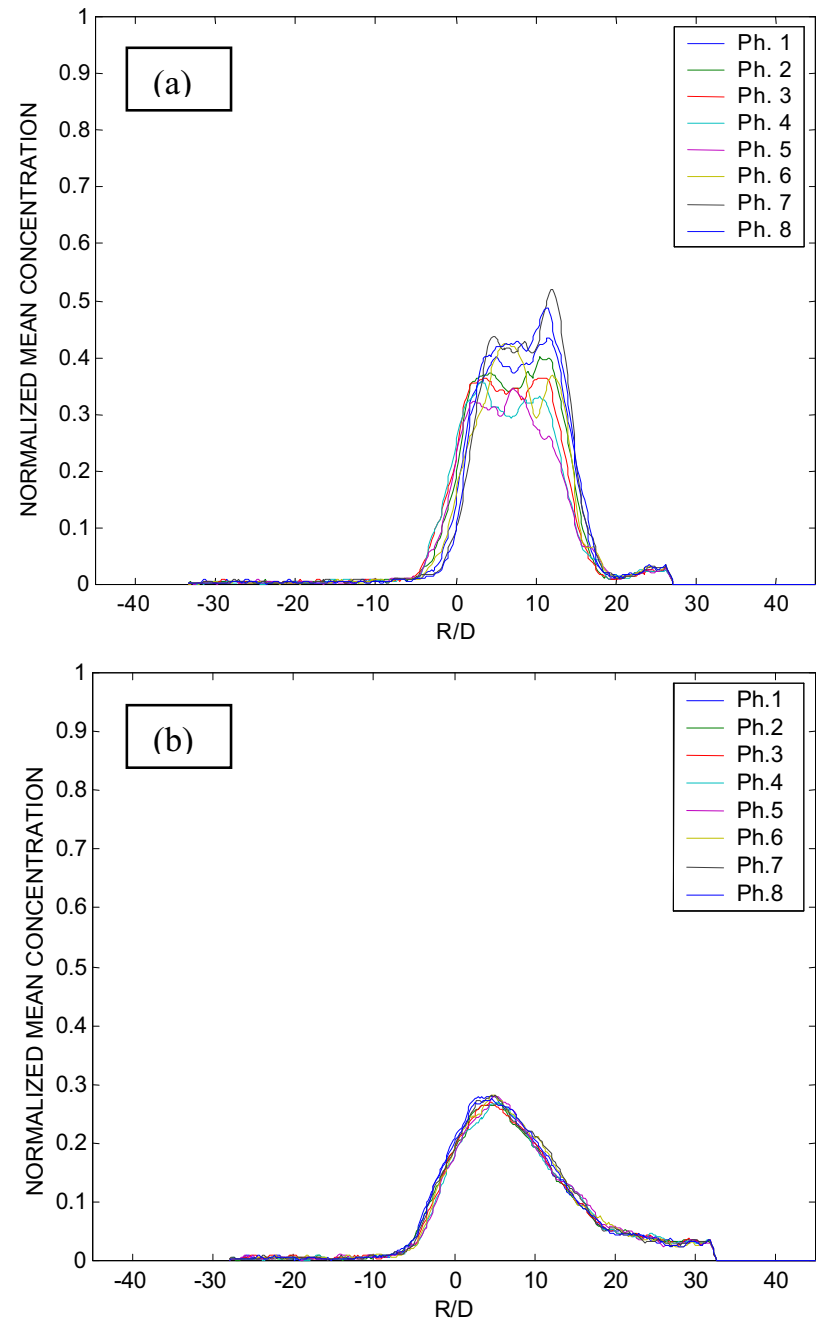

Figure 6. Mean concentration profiles, non-dimensionalized by the concentration in the jet origin, measured on the point of maximum height (a) and on the impact point (b), phase by phase; $\mathrm{Fr}=17.8, \mathrm{~T}=1.50 \mathrm{~s}, \mathrm{~A}=5.0 \mathrm{~mm}$.

Looking at Figure 9, the impact distance seems, differently from the maximum height, more biased by A: the curves of $\mathrm{A}=5.0 \mathrm{~mm}$ are generally closer to the stationary position if compared to the ones of $\mathrm{A}=12.5$ $\mathrm{mm}$. Moreover, when the values of $\mathrm{A}$ and $\mathrm{T}$ are both 
large, the effect of the wave on the INBJ is greater (red curves in Figure 9, for $\mathrm{T}=1.5 \mathrm{~s}$ and $\mathrm{A}=12.5 \mathrm{~mm}$ ). The only exception to this is the run with $\mathrm{Fr}=28.1, \mathrm{~T}=0.5 \mathrm{~s}$ and $\mathrm{A}=5.0 \mathrm{~mm}$ (blue line on Figure 9a): a physical explanation for this will be given in paragraph 3.3.
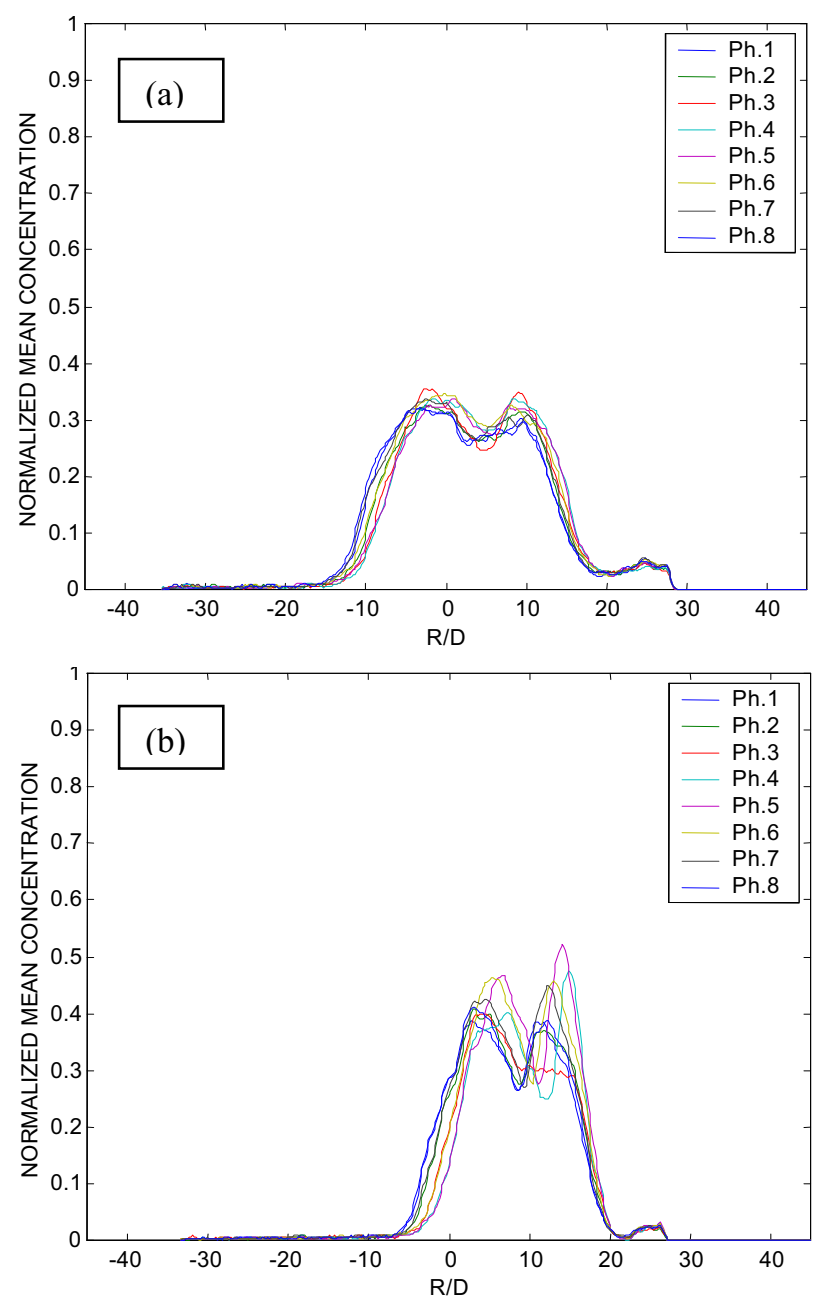

Figure 7. Mean concentration profiles, non-dimensionalized by the concentration in the jet origin, measured on the point of maximum height for two jets with $\mathrm{Fr}=28.1$ (a) and $\mathrm{Fr}=17.8$ (b), released in a wavy receiving body with $\mathrm{T}=1.05 \mathrm{~s}, \mathrm{~A}=12.5$ $\mathrm{mm}$, phase by phase.

The maximum height and the impact distance of an INBJ under a wave motion both decreases, showing a contraction of the INBJ: this contraction can be explained with the split into two branches of it (due to the periodic oscillation of the initial direction of the jet) that increases much the entrainment; on one side, the larger amount of external fluid entrained into the INBJ causes its premature braking, on the other side, the interaction between the two velocity fields (of the INBJ and of the wave motion) makes the interface between the jet and the external fluid more unstable, causing a larger dissipation of momentum.

Finally, a comparison between Figure 8a and 9a (for $\mathrm{Fr}=28.1$ ) and between Figure $8 \mathrm{~b}$ and $9 \mathrm{~b}($ for $\mathrm{Fr}=17.8$ ) makes it possible to spot how Fr bias INBJs: when Fr increases, the difference in density between the discharged and receiving fluid decreases and consequently the distance covered by the INBJ from the origin to the bottom increases (see the larger maximum heights and impact distances on Figures 9a and 11a when compared to the ones of Figures $9 \mathrm{~b}$ and $11 \mathrm{~b}$.
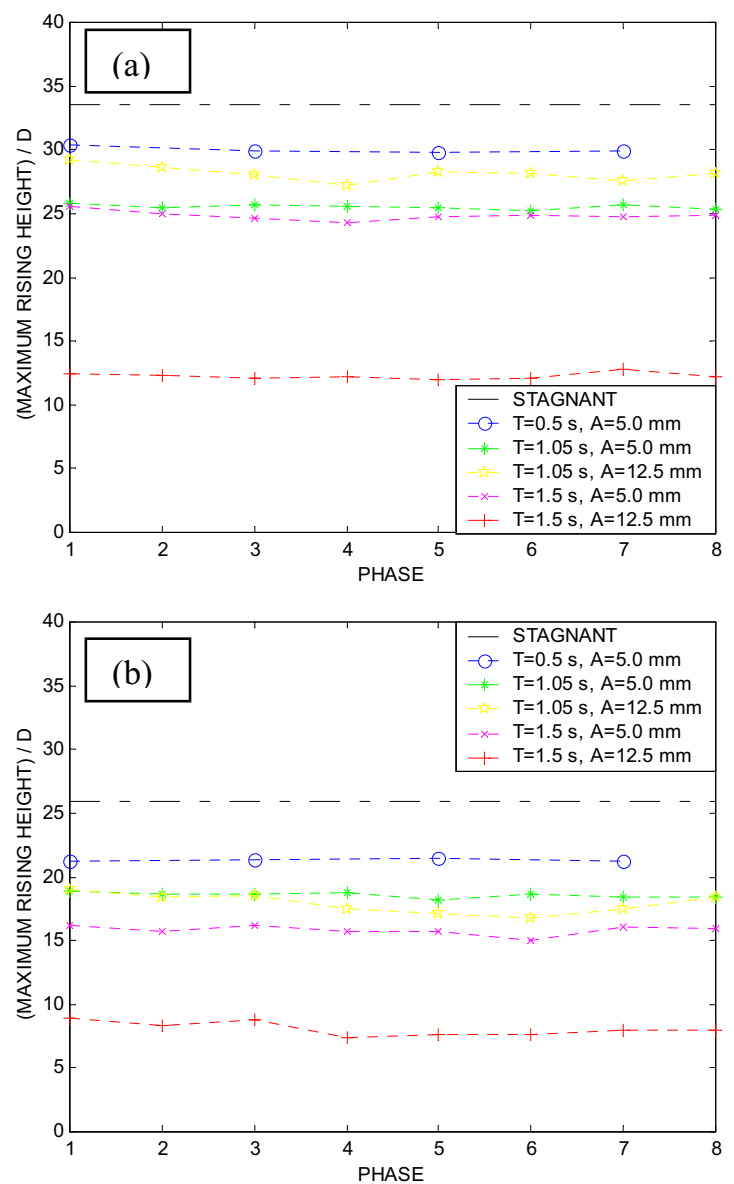

Figure 8. Maximum height of an INBJ in a stagnant environment (black dash-dotted line) and in wavy ones (coloured symbols connected by dotted lines) in the various phases, for $\mathrm{Fr}=28.1$ (a) and $\mathrm{Fr}=17.8(\mathrm{~b})$; for the run with $\mathrm{T}=$ $0.5 \mathrm{~s}$ and $\mathrm{A}=5.0 \mathrm{~mm}$, only four phases are plotted, as the oscillations are very small.

\subsection{Time averaged concentration fields}

In Figure $10(\mathrm{Fr}=28.1)$ and $10(\mathrm{Fr}=17.8)$ the time averaged concentration fields, computed for all the duration of each single run instead of phase by phase as above, are shown. Figures 10a and 11a are relative to the stagnant conditions, the others to the wavy conditions.

As highlighted by the bimodal distribution of the concentration fields, it is possible to verify that the interaction between an INBJ and a wave motion causes a splitting into two branches of the jet, one higher and one lower than the stationary position, that thereafter join together into a single branch. The oscillation of the initial region of the INBJ around the stationary position is more evident when A increases, as it is possible to see from the comparison of Figures $10 \mathrm{~d}$ and $10 \mathrm{f}(\mathrm{A}=12.5 \mathrm{~mm})$ with $10 \mathrm{~b}, 10 \mathrm{c}$ and $10 \mathrm{e}(\mathrm{A}=5.0 \mathrm{~mm})$ : in the first two Figures the bifurcation is more clearly visible, in the other three it is very weak. In fact, in Figure 10b the effect of the wave 
begins to be visible only very close to the point of maximum height, where the INBJ initial momentum has been largely dissipated; on Figure $10 \mathrm{~b}$ it is also possible to better understand the effect of the wave motion with $\mathrm{T}$ $=0.5 \mathrm{~s}$ and $\mathrm{A}=5.0 \mathrm{~mm}$ on the impact distance (compare with Figure 7a): the wave motion is too weak to slow the
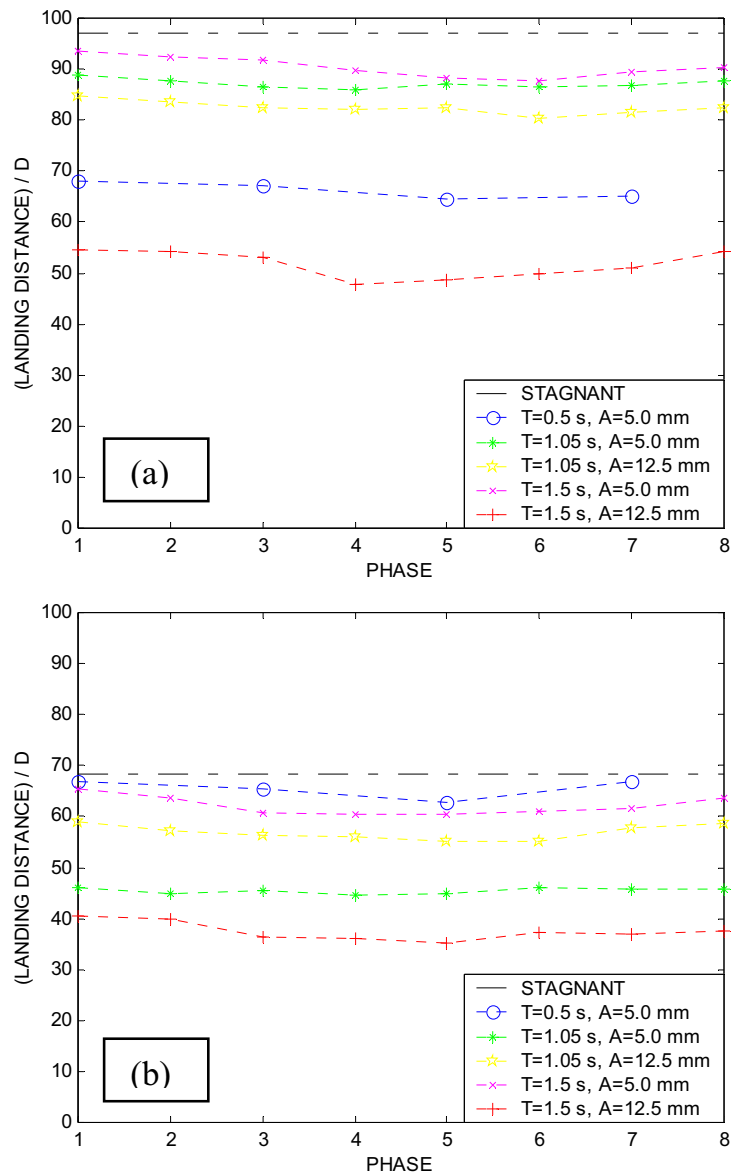

Figure 9. Impact distance of an INBJ in a stagnant environment (black dash-dotted line) and in wavy ones (coloured symbols connected by dotted lines) in the various phases, for $\mathrm{Fr}=28.1$ (a) and $\mathrm{Fr}=17.8$ (b); for the run with $\mathrm{T}=0.5 \mathrm{~s}$ and $\mathrm{A}=5.0 \mathrm{~mm}$, only four phases are plotted,as the oscillations are very small.

INBJ down enough, so the jet arrives close to the point of maximum height after having covered a distance long enough to dissipate its initial momentum and, consequently, it is mostly moved by the rotational movement of the wave. This means an additional mixing that makes the INBJ to fall down almost vertically. In Figure $10 \mathrm{c}$, for $\mathrm{T}=1.05 \mathrm{~s}$ and $\mathrm{A}=5.0 \mathrm{~mm}$, the oscillation caused by the wave motion is present (the INBJ widens more than in the stationary case) but not large enough to generate the bifurcation of the jet. Finally, in Figure 10e, for $\mathrm{T}=1.05 \mathrm{~s}$ and $\mathrm{A}=5.0 \mathrm{~mm}$, the initial bifurcation is weakened by the rotational movement imposed by the wave motion.

If now we focus on the INBJ in a wavy environment with $\mathrm{A}=12.5 \mathrm{~mm}$ (Figure 10d and 10f), it is now easier to guess how the splitting into two branches causes the contraction of the jet. Because of the strong deflection upwards and downwards imposed by the bifurcation, the jet have to spend a portion of its initial momentum to return to its initial direction and so it has not enough strength to reach the maximum height of the stationary case.

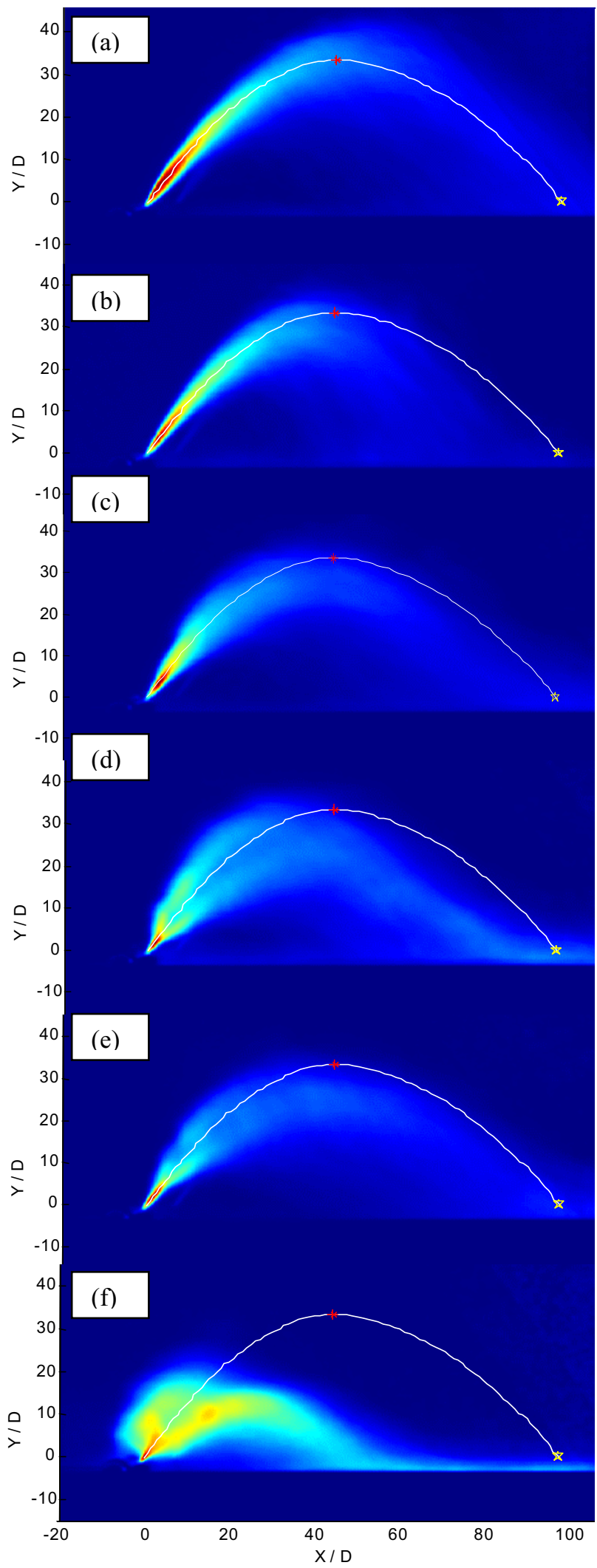

Figure 10. Time averaged concentration fields for an INBJ with $\mathrm{Fr}=28.1$, in a stagnant environment (a) and in wavy ones, with $\mathrm{T}=0.50 \mathrm{~s}$ and $\mathrm{A}=5.0 \mathrm{~mm}(\mathrm{~b}), \mathrm{T}=1.05 \mathrm{~s}$ and $\mathrm{A}=5.0 \mathrm{~mm}(\mathrm{c})$, $\mathrm{T}=1.05 \mathrm{~s}$ and $\mathrm{A}=12.5 \mathrm{~mm}(\mathrm{~d}), \mathrm{T}=1.50 \mathrm{~s}$ and $\mathrm{A}=5.0 \mathrm{~mm}(\mathrm{e})$ and $\mathrm{T}=1.50 \mathrm{~s}$ and $\mathrm{A}=12.5 \mathrm{~mm}(\mathrm{f})$. 
When, vice versa, the deflection is downwards, the effect on the maximum height is similar to the one of an INBJ released with a lower angle than the one used in these experiments, that is the jet to the horizontal, i.e. the jet reaches a lower maximum height (see Ferrari and Querzoli 2010 [17]). The bifurcation and the rotation imposed by the wave motion are the causes of the shorter impact distance: the fluid in the downward deflected branch is contrasted in its advance by the oscillation of the fluid trapped below it, while the fluid in the upward deflected branch would fall even closer if it does not met the lower branch, causing an additional mixing which slows the jet down. This is clearly visible in Figure 9f, on the point with $\mathrm{X} / \mathrm{D} \cong 15$ and $\mathrm{Y} / \mathrm{D} \cong 10$, with larger concentration.

Similar conclusions can be drawn from Figure 11, where the time averaged concentration fields are for $\mathrm{Fr}=$ 17.8. moreover, the comparison between Figures 10 and 11 confirms what previously stressed about the effect of Fr on INBJs: as Fr increases (i.e. the jets are heavier), INBs become smaller.

Summarizing, the major effect of $\mathrm{A}$ is to split the INBJ in two branches, while the one of $\mathrm{T}$ is more linked to the additional mixing, caused by a rotational motion, on the jet: they both contribute to dissipate the INBJ initial momentum and to increase its dilution. This because the bifurcation increases the exchange surface between the INBJ and the environment fluid (i.e., it increases the available surface for turbulent mixing), while the rotational motion can be seen as a mixing mechanism in addition to the entrainment (the only one available in a stagnant environment).

\subsection{Influence of the wave motion on the INBJ turbulent mixing}

In order to investigate the influence of wave motion on INBJs turbulent mixing, the variance of the concentration field has been computed: high values of the variance (colours close to the dark red in Figures 5, 12 and 13) point out regions where the changes in concentration are high and so the turbulent mixing is stronger; vice versa, low values of the variance (colours close to the dark blue) point out regions where the concentration tends to be constant and so the mixing is low or absent.

First of all, the variance of an INBJ in a stagnant environment (Figures 12a and 13a) will be introduced. An INBJ covers an initial distance from the outlet where it has a similar width to the initial diameter and the colour is close to the dark blue, pointing out that so far there is not yet strong turbulence.

Thereafter, the external boundaries of the INBJ suddenly experiences high variance values: this because the interface between the discharged fluid and the external one, characterized by the highest velocity and density gradients, becomes unstable, and the Kelvin-Helmoltz instabilities arise, causing a sudden widening because the consequent billows drag external fluid into the INBJ, causing mixing and dilution.

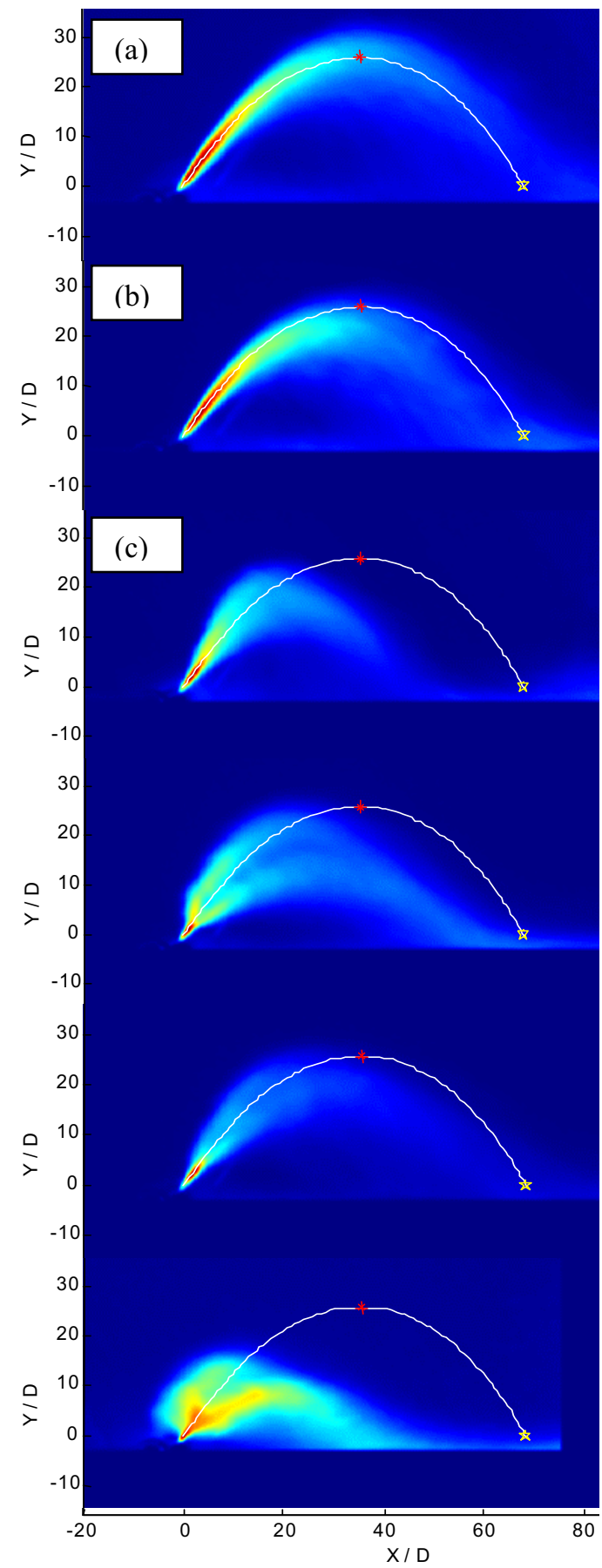

Figure 11. Time averaged concentration fields for an INBJ with $\mathrm{Fr}=17.8$, in a stagnant environment (a) and in wavy ones, with $\mathrm{T}=0.50 \mathrm{~s}$ and $\mathrm{A}=5.0 \mathrm{~mm}(\mathrm{~b}), \mathrm{T}=1.05 \mathrm{~s}$ and $\mathrm{A}=5.0 \mathrm{~mm}(\mathrm{c})$, $\mathrm{T}=1.05 \mathrm{~s}$ and $\mathrm{A}=12.5 \mathrm{~mm}(\mathrm{~d}), \mathrm{T}=1.50 \mathrm{~s}$ and $\mathrm{A}=5.0 \mathrm{~mm}(\mathrm{e})$ and $\mathrm{T}=1.50 \mathrm{~s}$ and $\mathrm{A}=12.5 \mathrm{~mm}(\mathrm{f})$.

Dilution grows along the INBJ trajectory, as it is possible to see from the comparison with figures 10a and 11a: the jet initial region has the highest concentration values and along the trajectory concentration decreases. As the buoyancy tends to anticipate the onset of these instabilities, as Fr decreases (buoyancy increases) the onset distance reduces, as shown in Ferrari and Querzoli 2010 [17]. In Figures 12a and 13a, the detachment of descending plumes is visible as well: they are the light blue lines beneath the INBJ, with a lower variance than the one of the Kelvin-Helmoltz billows. 


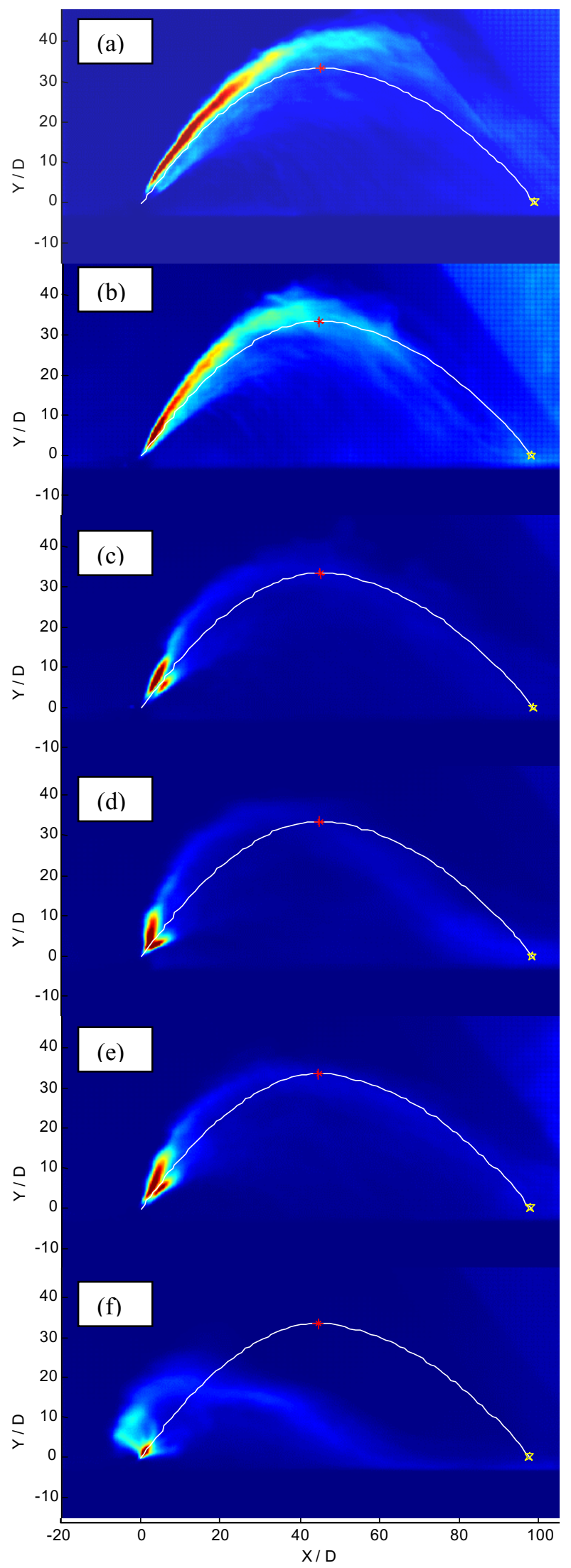

Figure 12. Variance of the concentration field of an INBJ with $\mathrm{Fr}=28.1$, in a stagnant environment (a) and in wavy ones, with $\mathrm{T}=0.50 \mathrm{~s}$ and $\mathrm{A}=5.0 \mathrm{~mm}(\mathrm{~b}), \mathrm{T}=1.05 \mathrm{~s}$ and $\mathrm{A}=5.0 \mathrm{~mm}(\mathrm{c})$, $\mathrm{T}=1.05 \mathrm{~s}$ and $\mathrm{A}=12.5 \mathrm{~mm}(\mathrm{~d}), \mathrm{T}=1.50 \mathrm{~s}$ and $\mathrm{A}=5.0 \mathrm{~mm}(\mathrm{e})$ and $\mathrm{T}=1.50 \mathrm{~s}$ and $\mathrm{A}=12.5 \mathrm{~mm}(\mathrm{f})$.

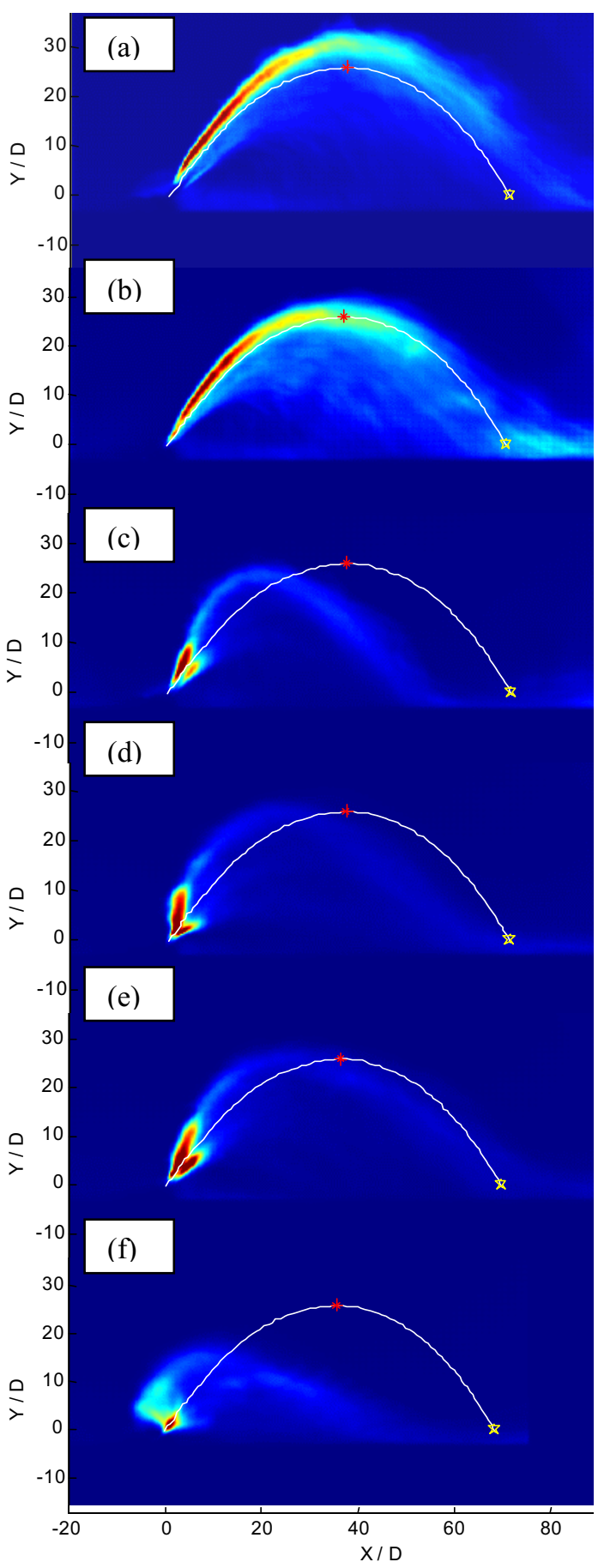

Figure 13. Variance of the concentration field of an INBJ with $\mathrm{Fr}=17.8$, in a stagnant environment (a) and in wavy ones, with $\mathrm{T}=0.50 \mathrm{~s}$ and $\mathrm{A}=5.0 \mathrm{~mm}(\mathrm{~b}), \mathrm{T}=1.05 \mathrm{~s}$ and $\mathrm{A}=5.0 \mathrm{~mm}(\mathrm{c})$, $\mathrm{T}=1.05 \mathrm{~s}$ and $\mathrm{A}=12.5 \mathrm{~mm}(\mathrm{~d}), \mathrm{T}=1.50 \mathrm{~s}$ and $\mathrm{A}=5.0 \mathrm{~mm}(\mathrm{e})$ and $\mathrm{T}=1.50 \mathrm{~s}$ and $\mathrm{A}=12.5 \mathrm{~mm}(\mathrm{f})$.

As a matter of fact, the turbulent structures, in the upper interface, tend to stay confined because there is a stable stratification and, consequently, strong concentration fluctuations; on the opposite, in the lower interface there is a stable stratification and the turbulent structures, when generated, tend to be dragged down by the negative buoyancy, generating a more spatially distributed turbulence with less intense concentration fluctuations. The inner region of the jet has lower value of variance for a longer space until it is reached by the intense turbulence generated at the INBJ boundaries, 
pointing out that the entrainment of external fluid has reached the whole jet. Even if the distance where the entrainment reaches the whole jet increases for increasing Fr, also the turbulent mixing length (i.e., the path available for mixing after the entrained fluid reaches the core of the jet) increases for increasing Fr, because the path followed by the jet before the impact point increases as well, as shown in [17].

In Figures from $12 \mathrm{~b}$ to $12 \mathrm{e}$ and from $13 \mathrm{~b}$ to $13 \mathrm{e}$ the variance of INBJs under different wave motions is shown. Apart from the runs of Figures $12 \mathrm{~b}$ and $14 \mathrm{~b}$, where $\mathrm{T}(0.5 \mathrm{~s})$ and $\mathrm{A}(5.0 \mathrm{~mm})$ are too small to generate the bifurcation of the jet, it is possible to see that the region of high variance begins, due to the oscillation imposed to the jet by the waves, soon after the outlet and extends along the two branches: for each change in direction the INBJ is in the same situation of its initial transitory: it must open its way in region of relatively still fluid. This anticipates the transition to turbulence, amplifies the turbulence and so increases the dilution. As already stated, the oscillation of the INBJ increases, compared to the stagnant case, the exchange interface between the discharged and the external fluid, allowing the turbulence to act on a larger surface: looking at Figure 5 , where the variance of the concentration field has been divided in 8 temporal phases, the number and the size of the high variance regions is larger than in the stagnant case. In Figure 5, in particular for the phases from 3 to 7 , it is possible to spot, for each phase, three regions of high variance: starting from the INBJ origin and moving along the axis, a first region is generated by the initial portion of the jet that, because of the wave induce oscillation in the JDR, impacts against an external environment where the velocity field is imposed by the wave motion and not by the jet (this mushroom-shaped high variance region is similar to the one in the front of a simple jet that, just released, try to open its way in the external environment). A second region is the one that, arisen from a previous oscillation, has in the meantime moved forward (the value of the variance in this second region, lower than the one of the first region, highlights that the velocities are now smaller and so the turbulence less intense). The third high variance region is the one detectable on the upper interface between the INBJ and the external environment, similar to the one previously described of the jet released in a still fluid.

\subsection{Influence of the wave motion on the INBJ dilution}

The effect of the increment of the number and size of the region with high variance (which is an increment of the surface available for the turbulent mixing) on the initial dilution of an INBJ will be described in this paragraph. The initial dilution is defined as the one reached by the INBJ when it falls down to the sea bottom, interacting with the biosphere: if this initial dilution is not high enough, many non-negligible environmental damages can occur (see Ferrari and Querzoli 2004 [18]). To better analyse the effect of a wave maotion on an INBJ, in Figure 14 the mean concentration profiles, non- dimensionalized by the initial concentrationand measured on the impact point, are shown in the case of stagnant environment (red line) and wavy one (blue line).
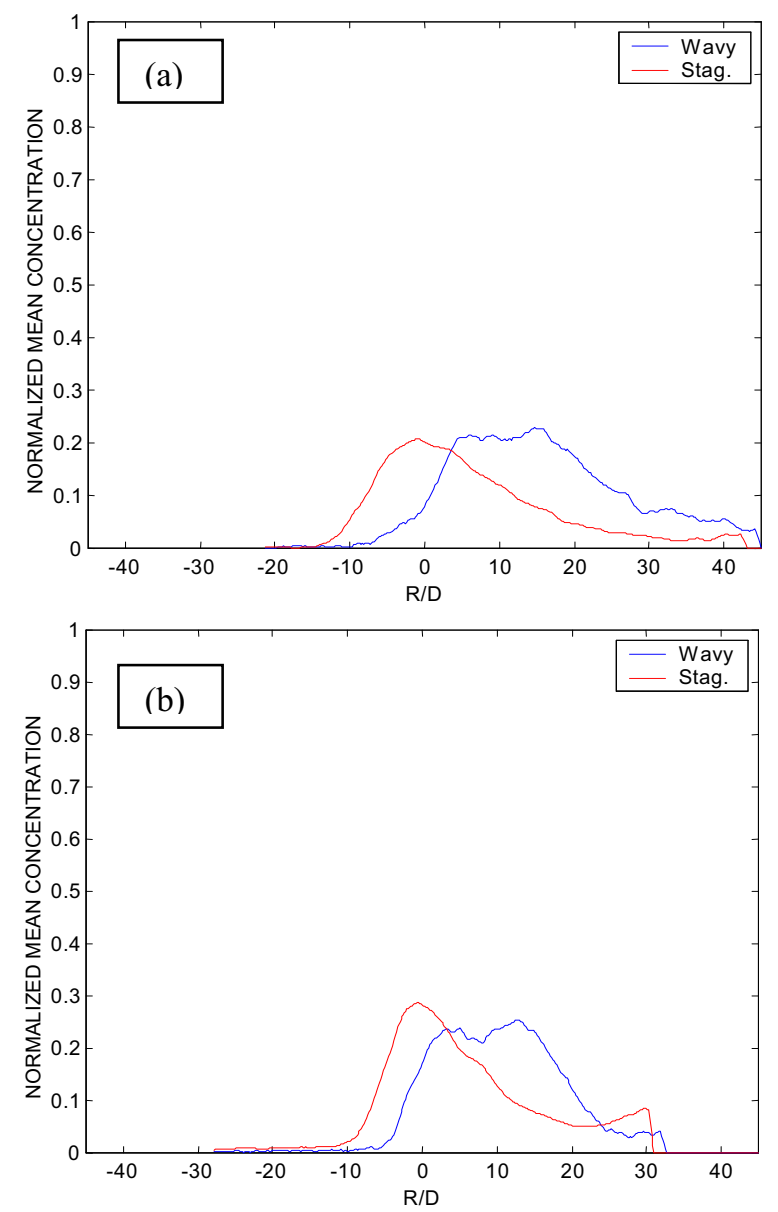

Figure 14. Mean concentration profiles, non-dimensionalized by the initial concentration, on the impact point, in a stagnant environment (red line) and in a wavy one (blue line) with $\mathrm{T}=$ $1.05 \mathrm{~s}$ and $\mathrm{A}=12.5 \mathrm{~mm}$, for two INBJs with $\mathrm{Fr}=28.1$ (a) and $\mathrm{Fr}=17.8(\mathrm{~b})$.

The two before mentioned concentration peaks are evident only on the blue line of Figure $14 \mathrm{~b}(\mathrm{Fr}=17.8)$ : this confirms that the bimodal distribution of the concentration is more pronounced for the lower Froude numbers. Moreover, the INBJ widening is larger in the case of wavy receiving body, whilst the reduction of the concentration peak is clearly visible only in the case of Figure $14 \mathrm{~b}$, relative to the heavier INBJ: this seems to imply that the effect of the wave motion on the jet dilution is bigger when the buoyancy increases. The larger widening of the INBJ and the reduction of the concentration peak point out that the dilution is larger when the INBJ is released in a wavy environment.

This increase in dilution can be explained by what previously stated: also if the region occupied by the INBJ discharged in a wavy environment tends to be smaller that the one occupied by the same jet discharged in a stagnant environment, the increment in number and size of the regions with high turbulence, caused by the wave motion, allow a larger exchange of fluid, an enhancement of the mixing and, consequently, the larger dilution measured on Figure 14. To get the most out of this 
mechanism and so to increase as much as possible the initial dilution, the wave period an amplitude must be large enough to force the split into two branches of the INBJ as soon as it surpasses the JDR.

\section{Conclusions}

The behavior of Inclined Negatively Buoyant Jets (INBJs) released into a wavy environment was analyzed from a statistical point of view, both considering the global phenomenon and its single phases. Analysis on the mean concentration fields allowed determining the deformations that the wave motion causes on INBJs: these, although being forced to oscillate, preserve their identity close to the origin, whilst further from their origin the INBJs are split into two separate branches that, eventually, fuse together before sinking to the bottom. The points of maximum height and the impact point (where the axis returns to the same level of the jet origin) do not rotate around the position they have in a stagnant environment, as it happens in vertical or horizontal simple jets, but always stay below it: the former is more biased by the wave period whilst the latter by the wave amplitude. This decrement increases as the wave period and amplitude increase and it is likely due to the interaction between two causes: one is the split into two branches of the central region of INBJs that, increasing the interchange surface between the jet and the external fluid, speeds up the dissipation of the initial momentum; the other is the rotational movement the waves impose to the central region of INBJs, that tends to slow its rising down. The splitting in two of INBJs under wave motion increases with low densimetric Froude numbers. Moreover, when Froude decreases, the concentration profiles, transversal to the jet axis, have a lower widening and tend to be more symmetrical: this is due to the larger mixing caused by the rotation, imposed by the wave motion on the descending branch of the INBJ, which contributes to spread more uniformly the discharged fluid. Analyses on variance and on the profile of concentration have confirmed that the wave motion increases the interface available for mixing and so allows amplifying the entrainment, extending the path available for mixing and, consequently, increasing the dilution.

\section{References}

1. L.A. Besalduch, M.G. Badas, S. Ferrari, G. Querzoli, European Physical Journal - Web of Conference, 67(02007), 02007-p.1-02007-p.6 (2014).

2. B. Jiang, A.W.K. Law, J.H.W. Lee, J. Hydr. Eng. 140(3), 241-253 (2014).

3. L.A. Besalduch, M.G. Badas, S. Ferrari, G. Querzoli, European Physical Journal - Web of Conference, 45(01012), 01012-p.1-01012-p.9 (2013).

4. C.J. Oliver, M.J. Davidson, R.I. Nokes, J.Hydr.Eng. 139(12), 1304-1308 (2013).

5. C.C.K. Lai, J.H.W. Lee, J. Hydro-env. Res. 6, 9-28 (2012)

6. D.A. Chin, J. Hydr. Eng. 113(8), 1006-1018 (1987).
7. Y. Ryu, K.A. Chang, N. Mori, J. Hydr. Eng. 131(12), 1088-1097 (2005).

8. K.A. Chang, Y. Ryu, N. Mori, J. Waterway, Port, Coastal, Ocean Eng., 135(3), 100-107 (2009).

9. S.C. Hsiao, T.W. Hsu, J.F. Lin, K.A. Chang, J. Waterway, Port, Coastal, Ocean Eng. 137(3), 109122 (2011).

10. D.A Chin, J. Waterway, Port, Coastal, Ocean Eng. 114(3), 331-345 (1988).

11. J.F. Lin, S.C. Hsiao, T.W. Hsu, K.A. Chang, J. Waterway, Port, Coastal, Ocean Eng. 139, 190-208 (2013).

12. H.H. Hwung, J.M. Chyan, J. Hydr. Res. 31(6), 791810 (1993).

13. M. Mossa, J. Hydr. Res. 42(1), 13-28 (2004).

14. J. Sharp J, Proceedings of the Institution of Civil Engineering, part 2, 81, 471-475 (1986).

15. J. Chyan, H. Hwung, Y. Chang, in Lee J., Cheung Y. (eds.), Environmental Hydraulics, Balkema (1991).

16. D.A. Chin, J. Hydr. Eng., 113(8), 1005-1017 (1987).

17. S. Ferrari, G. Querzoli, J. Hydr. Res. 48(5), 632-640 (2010).

18. S. Ferrari, G. Querzoli, in Avanzini C. (ed) MWWD \& IEMES 2004 - 3rd International Conference on Marine Waste Water Discharges and Marine Environment Proceedings, 27 September - 2 October 2004, Catania (Italy) (2004).

19. E.J. List, in: H.B. Fisher, E.J. List, R.C.Y. Koh, J. Imberger, N.H. Brooks (Eds), Mixing in inland and coastal water, New York, USA, Academic Press, 315-389 (1979). 